\title{
A permutation representation that knows what "Eulerian" means
}

\author{
Roberto Mantaci ${ }^{1}$ and Fanja Rakotondrajao ${ }^{2 \dagger}$ \\ ${ }^{1}$ LIAFA, Université Paris 7 - Denis Diderot, e-mail: mantaci@liafa.jussieu.fr \\ ${ }^{2}$ Département de Mathématiques, Faculté des Sciences, Université d'Antananarivo, \\ e-mail: frakoton@syfed.refer.mg
}

received Feb 5, 2001, revised May 14, 2001, accepted May 14, 2001.

\begin{abstract}
Eulerian numbers (and "Alternate Eulerian numbers") are often interpreted as distributions of statistics defined over the Symmetric group. The main purpose of this paper is to define a way to represent permutations that provides some other combinatorial interpretations of these numbers. This representation uses a one-to-one correspondence between permutations and the so-called subexceedant functions.
\end{abstract}

Keywords: permutation statistics, Eulerian numbers, subexceedant functions

\section{Introduction}

Several works in discrete mathematics and theoretical computer science deal with effective ways to represent permutations.

A pioneer of this matter is Lehmer [6] who associates bijectively with each permutation over the interval $[n]=\{1,2, \ldots, n\}$ a function $f:[n] \mapsto\{0,1, \ldots, n-1\}$. There are several ways to establish this one-to-one correspondence but perhaps the most classical of them is the so-called Lehmer code (or inversion table). The Lehmer code of a permutation $\sigma$ is a function $f$ defined in the following way, for all $i \in[n]$ :

$$
f(i) \text { is the number of indices } j \text { such that } 1 \leq j<i \text { and } \sigma(j)<\sigma(i) \text {. }
$$

The description of the Lehmer code can also be found in Knuth [5]. However, Knuth cites Marshall Hall's observation [4] stating that this function uniquely determines the corresponding permutation.

These functions have obviously the following property :

$$
0 \leq f(i)<i \text { for all } 1 \leq i \leq n,
$$

and because of this property such functions are called subexceedant, a term that can be found for instance in the work of Dumont and Viennot [2].

Subexceedant functions are certainly an effective coding for permutations, because the word $f(1) f(2) \ldots f(n)$ for a subexceedant function can be represented more effectively than the word $\sigma(1) \sigma(2) \ldots \sigma(n)$ of the corresponding permutation. Firstly, the word representing $f$ is generally a word on a smaller alphabet than $\{1,2, \ldots, n\}$, which may allow to code the letters of the alphabet with shorter code words. Moreover, a word of length $n$ on an alphabet of cardinality smaller than $n$ is likely to contain several consecutive occurrences of a same integer, which notoriously allows an even more compact representation of the word itself. For instance, if a word representing a subexceedant function $f$ contains 50 consecutive occurrences of an integer $i$, it is certainly more efficient to code that factor of the word $f$ with the couple $(50, i)$ than with 50 consecutive $i$ 's.

${ }^{\dagger}$ This author was supported by University of Paris 7 - Denis Diderot during her staying at LIAFA as invited Maître de Conférence 
We present here a way to associate with every permutation a map $f:[n] \mapsto[n]$ such that

$$
1 \leq f(i) \leq i \text { for all } 1 \leq i \leq n .
$$

For obvious reasons, we will still call these function subexceedant, although for sake of consistency with previous litterature they would be better called with the barbarian term non strictly subexceedant functions.

Besides the advantage of compactness that we have already mentioned, our coding seems to be appropriate to transfer to the subexceedant functions some properties that are typical of permutations, namely the distribution of certain statistics called Eulerian.

Eulerian numbers $A_{n, k}$ with $n \geq 1$ and $1 \leq k \leq n$, are "a classic" in enumerative combinatorics. Their properties are described in details in most introductory manuals of enumerative combinatorics such as Riordan [10]. A further, thorough investigation of these numbers is in the fundamental work of Foata and Schützenberger [3], while applications of these numbers to analysis of algorithms (namely, sorting algorithms) can be found in Knuth [5] and applications to combinatorics of words can be found in Lothaire [7].

Eulerian numbers are often studied by seeing them as distributions of statitics (therefore called Eulerian statistics) defined over permutations, such as descents, excedances, readings.

In this paper we look at some statistics defined over the set of subexceedant functions and show that they are also Eulerian using the coding that we have introduced.

A natural extension of this work would be to study the distribution of sequences of consecutive occurrences of a same integer in a random subexceedant function, in order to establish when it is convenient to use the corresponding subexceedant function to represent a permutation in a more effective fashion.

However, the correspondence between certain properties of the Eulerian statistics over the permutations and the properties of the Eulerian statistics over subexceedant functions is already an encouraging result in itself.

We also show that other properties of permutations can be "read" over the corresponding subexceedant function. In particular, we show that it is possible to characterize subexceedant functions associated with even permutations or those associated with derangements.

\section{Notations, definitions and preliminaries}

Let us denote by $[n]$ the interval $\{1,2,3, \ldots, n\}$ and by $\sigma$ a permutation of the symmetric group $\mathfrak{S}_{n}$. We will adhere to the following convention: when we multiply two permutations, the leftmost permutation always acts first, for example if $\sigma=(13)(245)$ and $\tau=(1543)(2)$ then $\sigma \tau=(1)(235)(4)$.

Definition 2.1 An integer $i \in[n]$ is a fixed point for $\sigma$ if $\sigma(i)=i$.

Definition 2.2 A pernutation $\sigma$ is a derangement if $\sigma$ has no fixed points.

We will denote by $\mathcal{D}_{n}$ the set of all derangements in $\mathfrak{S}_{n}$ and by $d_{n}$ the cardinality of $\mathcal{D}_{n}$.

Definition 2.3 A subexceedant function $f$ on $[n]$ is a map $f:[n] \mapsto[n]$ such that

$$
1 \leq f(i) \leq \text { ifor all } 1 \leq i \leq n .
$$

The authors were introduced to the subject of subexceedant functions by D. Dumont [i]]. We will denote by $\mathcal{F}_{n}$ the set of all subexceedant functions on $[n]$, and we will represent a subexceedant function $f$ over $[n]$ by the word $f(1) f(2) \cdots f(n)$.

Example 2.4 The following are the sets $\mathcal{F}_{n}$ for $n=1,2,3$ :

$$
\begin{aligned}
& \mathcal{F}_{1}=\{1\} \\
& \mathcal{F}_{2}=\{11,12\} \\
& \mathcal{F}_{3}=\{111,112,113,121,122,123\}
\end{aligned}
$$

It is easy to verify that $\operatorname{card} \mathcal{F}_{n}=n !$, since from each subexceedant $f$ over $[n-1]$, one can obtain $n$ distinct subexceedant functions over $[n]$ by adding any integer $i \in[n]$ at the end of the word representing $f$.

We will denote by $\operatorname{Im}(f)$ the set of the elements in $f([n])$ and by $\operatorname{IMA}(f)$ the cardinality of this set.

Example 2.5 Let $f=112352$ be a subexceedant function on $\{1,2, \cdots, 6\}$, then $\operatorname{Im}(f)=\{1,2,3,5\}$ and $\operatorname{IMA}(f)=$ 4. 


\section{A bijection between the subexceedant functions and permutations}

We will give a bijection between $\mathfrak{S}_{n}$ and $\mathcal{F}_{n}$ and highlight some of its properties.

Let $\phi: \mathcal{F}_{n} \mapsto \mathfrak{S}_{n}$ be the map which associates with the subexceedant function $f$ the permutation $\sigma_{f}=\phi(f)$ defined as a product of transpositions by:

$$
\sigma_{f}=(1 f(1))(2 f(2)) \cdots(n f(n))
$$

Notice that there is an abuse of notation in the definition of $\sigma_{f}$. Indeed, if $f(i)=i$, then the cycle $(i f(i))=(i)$ does not really denote a transposition but simply the identty permutation.

Proposition 3.1 The map $\phi$ is a bijection from $\mathcal{F}_{n}$ onto $\mathfrak{S}_{n}$.

Proof. Since $\mathfrak{S}_{n}$ and $\mathcal{F}_{n}$ both have cardinality $n$ !, it suffices to prove that $\phi$ is injective. Let $f$ and $g$ be two subexceedant functions on $[n]$. Assume that $\phi(f)=\phi(g)$ i.e. $\sigma_{f}=\sigma_{g}$. So we have:

$$
(1 f(1))(2 f(2)) \cdots(n f(n))=(1 g(1))(2 g(2)) \cdots(n g(n)) \text {. }
$$

Since $\sigma_{f}=\sigma_{g}$, then in particular $\sigma_{f}(n)=\sigma_{g}(n)$, but by definition $\sigma_{f}(n)=f(n)$ and $\sigma_{g}(n)=g(n)$, so $f(n)$ and $g(n)$ are equal as well. Let us multiply both members of this equality on the right by the permutation $(n f(n))=(n g(n))$, we obtain:

$$
(1 f(1))(2 f(2)) \cdots(n-1 f(n-1))=(1 g(1))(2 g(2)) \cdots(n-1 g(n-1)) .
$$

Now, if we apply the same process to these two permutations, we obtain $f(n-1)=g(n-1)$. By iterating, we can conclude that $f(i)=g(i)$ for all integers $i$ and then that $f=g$.

Example 3.2 Take $f=112435487$ and $n=9$. The permutation $\sigma_{f}=\phi(f)$ is:

$$
\begin{aligned}
& \sigma_{f}=(1)(21)(32)(4)(53)(65)(74)(8)(97) \\
& =(16532)(497)(8)
\end{aligned}
$$

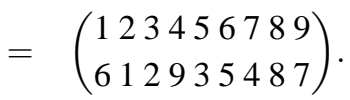

Let $\sigma$ be a permutation of the symmetric group $\mathfrak{S}_{n}$ and $f$ be the inverse image of $\sigma$ by $\phi$. Then $f$ can be constructed as below:

1. Set $f(n)=\sigma(n)$.

2. Multiply $\sigma$ on the right by the transposition $(n \sigma(n))$ (this operation consists in exchanging the image of $n$ and the image of $\sigma^{-1}(n)$ ), we obtain this way a new permutation $\sigma_{1}$ having $n$ as a fixed point. Thus $\sigma_{1}$ can be considered as a permutation of $\mathfrak{S}_{n-1}$. Then set $f(n-1)=\sigma_{1}(n-1)$.

3. Apply now the same process to the permutation $\sigma_{1}$, by multiplying it by $\left(n-1 \sigma_{1}(n-1)\right)$ and obtain this way $f(n-2)$. By iterating, it is possible to obtain all values of $f(i)$ for all integers $i$.

Example 3.3 Let us consider the permutation

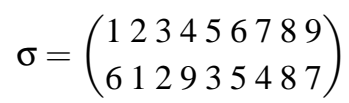

defined in the previous example. We are going to construct the subexceedant function $f$ that is the inverse image of $\sigma$ by the bijection $\phi$. First, we have $f(9)=\sigma(9)=7$. To compute $f(8)$, we have to exchange the images of 9 and $\sigma^{-1}(9)=4$ in $\sigma$ and we obtain the permutation

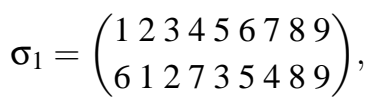


which is indeed a permutation of $\mathfrak{S}_{8}$ and hence that can be considered as

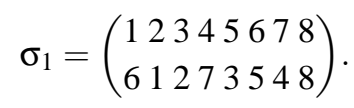

Then we have $f(8)=\sigma_{1}(8)=8$. To define $f(7)$, we have to exchange the images of 8 and $\sigma_{1}{ }^{-1}(8)=8$ in $\sigma_{1}$ and we obtain the permutation

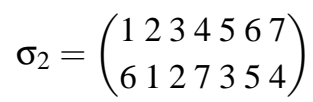

in $\mathfrak{S}_{7}$, then $f(7)=\sigma_{2}(7)=4$. By using the same process, we obtain the following permutations and the different values of $f(i)$ :

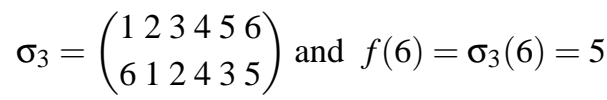

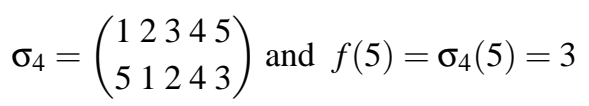

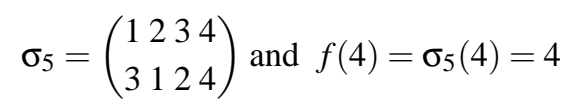

$$
\begin{aligned}
& \sigma_{6}=\left(\begin{array}{lll}
1 & 2 & 3 \\
3 & 1 & 2
\end{array}\right) \text { and } f(3)=\sigma_{6}(3)=2 \\
& \sigma_{7}=\left(\begin{array}{ll}
1 & 2 \\
2 & 1
\end{array}\right) \text { and } f(2)=\sigma_{7}(2)=1 \\
& \sigma_{8}=\left(\begin{array}{l}
1 \\
1
\end{array}\right) \text { and } f(1)=\sigma_{8}(1)=1 .
\end{aligned}
$$

Finally, we obtain the subexceedant function $f=112435487$.

D. Dumont [I] noticed that the cardinality of the set $\left\{f \in \mathcal{F}_{n} \mid \operatorname{IMA}(f)=k\right\}$ is the Eulerian number $A_{n, k}$. This result can be proven in different ways. One possibility is to show that the numbers $s_{n, k}=\operatorname{card}\left\{f \in \mathcal{F}_{n} \mid \operatorname{IMA}(f)=k\right\}$ satisfy the recursive relation of the Eulerian numbers $A_{n, k}$, i.e.

$$
s_{n, k}=(n-k+1) s_{n-1, k-1}+k s_{n-1, k} .
$$

This is not hard to prove, since all the elements of the set $\left\{f \in \mathcal{F}_{n} \mid \operatorname{IMA}(f)=k\right\}$ are obtained in a unique way

- either from an element $f$ of $\mathcal{F}_{n-1}$ with $\operatorname{IMA}(f)=k$ by concatenating at its end one of the integers in the set $\operatorname{Im}(f)$

- or from an element $f$ of $\mathcal{F}_{n-1}$ with $\operatorname{IMA}(f)=k-1$ by concatenating at its end one of the $n-(k-1)$ integers in the set $[n] \backslash \operatorname{Im}(f)$.

In order to give a bijective proof of this result, we recall a definition.

Definition 3.4 A pemutation $\sigma$ has an anti-excedance in $i \in[n]$ if $\sigma(i) \leq i$.

We denote by $\operatorname{AX}(\sigma)$ the number of the anti-excedances of $\sigma$. The Eulerian number $A_{n, k}$ equals the cardinality of the set $\left\{\sigma \in \mathfrak{S}_{n} \mid \sigma\right.$ has $k$ anti-excedances $\}$ (see [3], [8], [9]).

We now prove that $s_{n, k}=A_{n, k}$ by showing that an appropriate restriction of the map $\phi$ defined in Prop. 3.1 is indeed a bijection between the set $\left\{\sigma \in \mathfrak{S}_{n} \mid \mathrm{AX}(\sigma)=k\right\}$ and the set $\left\{f \in \mathcal{F}_{n} \mid \operatorname{IMA}(f)=k\right\}$.

Proposition 3.5 Let $f$ be a subexceedant function of the set $\mathcal{F}_{n}$ and let $\sigma_{f}=\phi(f)$ be the image of $f$ by the bijection $\phi$. The two following assertions are equivalent:

1. $i \in \operatorname{Im}(f)$ 
2. $\sigma_{f}$ has an anti-excedance at $\sigma_{f}^{-1}(i)$

Therefore, the number of images of $f$ is equal to the number of anti-excedances of $\sigma_{f}$.

Proof. Assume that $i \in \operatorname{Im}(f)$ and let $\left\{j_{1}, j_{2}, \cdots, j_{\ell}\right\}$ be the set of integers such that $f\left(j_{k}\right)=i$. The integer $j_{k}$ are all greater than or equal to $i$ since $f$ is a subexceedant function. Then the transpositions $\left(j_{1} i\right),\left(j_{2} i\right), \cdots,\left(j_{\ell} i\right)$ appear in this order in the definition of $\sigma_{f}$, therefore the image of $j_{\ell}$ by $\sigma_{f}$ is necessarily $i$ because the integer $j_{\ell}$ never appears in the product before the occurrence of $\left(j_{\ell} i\right)$ (since $f$ is subexceedant) and $i$ never appears in this product after the occurrence of $\left(j_{\ell} i\right)$ since $j_{\ell}$ is the largest element having $i$ as image. So $j_{\ell}=\sigma_{f}{ }^{-1}(i)$ and hence $\sigma_{f}\left(j_{\ell}\right)=\sigma_{f}\left(\sigma_{f}^{-1}(i)\right)=i$ with $i<j_{\ell}$, that is, $\sigma_{f}$ has an anti-excedance in $j_{\ell}=\sigma_{f}^{-1}(i)$.

Conversely, let us take an element $i \notin \operatorname{Im}(f)$ and let us look at the product of transpositions that defines $\sigma_{f}$. The integer $i$ only appears once, exactly in the transposition $(i f(i))$ with $f(i)<i$. Then the inverse image of $i$ is either $f(i)$ itself or an integer that appears in a transposition that occurs on the left of $(i f(i))$ (notice that all transpositions that appear on the left of $(i f(i))$ involve only integers that are smaller than $i)$. In either case, the inverse image of $i$ is an integer smaller than $i$, that is, $\sigma_{f}^{-1}(i)<i=\sigma_{f}\left(\sigma_{f}^{-1}(i)\right)$ and hence $\sigma_{f}$ has an excedance in $\sigma_{f}^{-1}(i)$. This concludes the proof.

\subsection{Derangements and subexceedant functions}

Now, we are going to establish that derangements or permutations without fixed points are in bijection with a precise class of subexceedant functions.

Definition 3.6 Let $f$ a subexceedant function and $i$ an integer in [n]. We say that $i$ is a multiple fixed point for $f$ if:

1. $f(i)=i$ and

2. there exists an integer $j>i$ such that $f(j)=i$.

Example 3.7 Take $f=12133$. The integer 1 is a multiple fixed point, whereas 2 and 3 are not.

Proposition 3.8 Let $\mathcal{F}_{n}$ be the set of subexceedant functions, $\mathfrak{S}_{n}$ the symmetric group and $\phi$ the bijection between $\mathcal{F}_{n}$ and $\mathfrak{S}_{n}$ described in Prop. 3.1. Let $f$ be a subexceedant function and $\sigma_{f}=\phi(f)$ the corresponding permutation. Then $\sigma_{f}$ is a derangement if and only if all fixed points of $f$ are multiple.

Proof. Let $f$ be a subexceedant function and $i$ be a non multiple fixed point. In this case, $f(i)=i$ and $f(j) \neq i$ for all integers $j \neq i$. Then the integer $i$ appears only once in the transposition product defining $\sigma_{f}$ : this unique occurrence is the "transposition" $(i f(i))$, which is the identity permutation. Therefore $\sigma_{f}(i)=i$, that is, $i$ is a fixed point for $\sigma_{f}$ and $\sigma_{f}$ is not a derangement.

Conversely, if $\sigma_{f}$ is not a derangement and an integer $i$ is a fixed point for $\sigma$ then by using the inverse construction of $\phi$, we have $f(i)=i$ and there is not another integer $j \neq i$ such that $f(j)=i$. This concludes the proof.

\section{Other interpretations of the numbers $P_{n, k}$ and $D_{n, k}$}

We will denote respectively by $D_{n, k}$ and $P_{n, k}$ the cardinalities of the set of all odd permutations in $\mathfrak{S}_{n}$ having $k$ antiexcedances and the set of all even permutations in $\mathfrak{S}_{n}$ having $k$ anti-excedances. These numbers were introduced by the first author in [8] and further studied in [9]. The numbers $P_{n, k}$ are also referred to as "Alternate Eulerian numbers". We recall that the numbers $P_{n, k}$ and $D_{n, k}$ satisfy the recursive formulas:

$$
\begin{aligned}
& P_{n, k}=(n-k) D_{n-1, k-1}+k D_{n-1, k}+P_{n-1, k-1} \\
& D_{n, k}=(n-k) P_{n-1, k-1}+k P_{n-1, k}+D_{n-1, k-1}
\end{aligned}
$$

for all positive integers $n$ and $1 \leq k \leq n$. We will study in this section two other interpretations of these numbers.

Proposition 4.1 Let $f \in \mathcal{F}_{n}$ be a subexceedant function and $\sigma_{f}=\phi(f)$ be the corresponding permutation. Then $\sigma_{f}$ is an even permutation if and only if the number $a(f)$ of strict anti-excedances of $f$ is even. 
Proof. Notice that $f$ has a strict anti-excedance in $i$ if and only if the integer $i$ is not a fixed point for $f$. Then in the transpositions product defining the permutation $\sigma_{f}$, i.e. in the decomposition $\sigma_{f}=(1 f(1)) \cdots(n f(n))$, the number of the cycles that are not equal to the identity coincide with the number of strict anti-excedances of $f$. The parity of a permutation $\sigma$ is the same as the parity of the number of transpositions that occur in any product of transposition that is equal to $\sigma$.

Corollary 4.2 The number $P_{n, k}$ equals the cardinality of the set of all subexceedant functions in $\mathcal{F}_{n}$ such that the number of the strict anti-excedances of $f$ is even and $\operatorname{IMA}(f)=k$, and the number $D_{n, k}$ designates the cardinality of the set of all subexceedant functions in $\mathcal{F}_{n}$ such that the number of the strict anti-excedances of $f$ is odd and $\operatorname{IMA}(f)=k$.

We give another combinatorial interpretation of the number $P_{n, k}$ related to subexceedant functions. We show that subexceedant functions having image of cardinality $k$ and with an even number of strict anti-excedances are in bijection with subexceedant functions having image of cardinality $k$ with an odd number of occurrences of 1 .

Definition 4.3 A subexceedant function $f$ has a strict anti-excedance in $i$ if $f(i)<i$.

We will denote by $a(f)$ the number of the strict anti-excedances of $f$ and by $b(f)$ the number of occurrences of the integer 1 in $f$.

Example 4.4 Take $n=6$ and $f=112433$, then $a(f)=4$ and $b(f)=2$.

Definition 4.5 Define the map $\psi: \mathcal{F}_{n} \mapsto \mathcal{F}_{n}$ as follows:

- if $a(f)+b(f)$ is odd, then $\psi(f)=f$ and

- if $a(f)+b(f)$ is even, then look for the smallest integer i that satisfies either one of the following properties:

$\left(p_{1}\right) i \in \operatorname{Im}(f) \backslash\{1\}$ and $f(i)=1$, or

$\left(p_{2}\right) i \in \operatorname{Im}(f) \backslash\{1\}$ and $i$ is a multiple fixed point.

Then $\psi(f)$ is the subexceedant function described as follows:

- if $i$ satisfies $\left(p_{1}\right)$, then we define $\psi(f)(j)=f(j)$ for all $j \neq i$ and $\psi(f)(i)=i$.

- if i satisfies $\left(p_{2}\right)$, then we define $\psi(f)(j)=f(j)$ for all $j \neq i$ and $\psi(f)(i)=1$.

Lemma 4.6 The map $\psi$ is well defined, indeed, if $a(f)+b(f)$ is even, then there always exists an integer $i$ which satisfies either property $\left(p_{1}\right)$ or property $\left(p_{2}\right)$.

Proof. We will prove this result by contradiction. Let $1, i_{2}, \cdots, i_{k}$ be the elements of $\operatorname{Im}(f)$ and suppose that $f(i) \neq 1$ for all integers $i$ in $\operatorname{Im}(f) \backslash\{1\}$ and also that none of the integers in $\operatorname{Im}(f) \backslash\{1\}$ is a multiple fixed point.

If $j$ is an integer such that $1<j<i_{2}$, then necessarily $f(j)=1$ because $f$ is subexceedant and the next smallest possible value for $f(j)$ is $i_{2}$, with $i_{2}>j$, which would contradict the fact that $f$ is subexceedant. The image of $i_{2}$ is clearly $i_{2}$ itself because we suppose that $f\left(i_{t}\right) \neq 1$ for all $t=2, \cdots, k$. If $j$ is an integer such that $i_{2}<j<i_{3}$, then necessarily $f(j)=1$ indeed $f$ is subexceedant and therefore $f(j)$ can only be 1 or $i_{2}$, but if $f(j)=i_{2}$ then $i_{2}$ would be a multiple fixed point. Analogously we prove that $f\left(i_{t}\right)=i_{t}$ for all $t=2, \cdots, k$ and that $f(j)=1$ for all integers $j \notin \operatorname{Im}(f)$. Then $f$ has exactly $n-k+1$ occurrences of 1 (all elements, except $i_{2}, \cdots, i_{k}$, have image 1 ) and exactly $n-k$ strict anti-excedances ( $f$ presents a strict anti-excedance in all $j \notin \operatorname{Im}(f)$ ). This is a contradiction since, by hypothesis, these two numbers must have the same parity.

Example 4.7 This example shows how the map $\psi$ works.

- Let $f_{1}$ be the subexceedant function 112413. Then $a\left(f_{1}\right)=4$ and $b\left(f_{1}\right)=3$. Since $a\left(f_{1}\right)+b\left(f_{1}\right)=7$ which is an odd number, then $\psi\left(f_{1}\right)=f_{1}$. 
- Let $f_{2}$ be the subexceedant function 112433. Then $a\left(f_{2}\right)=4$ and $b\left(f_{2}\right)=2$, then $a\left(f_{2}\right)+b\left(f_{2}\right)=6$ which is an even number, therefore, in order to compute $\psi\left(f_{2}\right)$ we need to determine the smallest integer $i$ in $\operatorname{Im}\left(f_{2}\right) \backslash\{1\}=\{2,3,4\}$ such that either $f_{2}(i)=1$, or $i$ is a multiple fixed point. This integer is 2 , which satisfies the first of these properties. The function $\psi\left(f_{2}\right)$ is then obtained from $f_{2}$ by making $i=2$ a multiple fixed point (that is, by setting $f_{2}(2)=2$ ). Hence, $\psi\left(f_{2}\right)=122433$.

- Let $f_{3}$ be the subexceedant function 122433. Then $a\left(f_{3}\right)=3$ and $b\left(f_{3}\right)=1$, then $a\left(f_{3}\right)+b\left(f_{3}\right)=4$ which is an even number, therefore, in order to compute $\psi\left(f_{3}\right)$ we need to determine the smallest integer i in $\operatorname{Im}\left(f_{3}\right) \backslash\{1\}=\{2,3,4\}$ such that either $f_{3}(i)=1$, or $i$ is a multiple fixed point. This integer is 2 , which satisfies the second of these properties. The function $\psi\left(f_{3}\right)$ is then obtained from $f_{2}$ by setting $\left.f_{3}(2)=1\right)$. Hence, $\psi\left(f_{2}\right)=112433$.

Remark 4.8 For all subexceedant functions $f$ in $\mathcal{F}_{n}$ one has $\operatorname{Im}(f)=\operatorname{Im}(\psi(f))$.

Proposition 4.9 The map $\psi$ is a bijection between the set of the subexceedant functions in $\mathcal{F}_{n}$

Proof. It follows from the definition of $\psi$ that for all $f$ in the set of the subexceedant functions, one has

$$
\psi(\psi(f))=f
$$

and hence $\psi$ is invertible (and its inverse is itself).

Proposition 4.10 The map $\psi$ associates a subexceedant function $f$ having an even (respectively, odd) number of occurrences of 1 , with a subexceedant function $\psi(f)$ having an odd (respectively, even) number of strict antiexcedances.

Proof. Consider the two following cases.

1. If $a(f)+b(f)$ is odd, then $\psi(f)=f$ and the statement is obvious.

2. If $a(f)+b(f)$ is even, then

(a) either $f$ has an even number of strict anti-excedances and the number of occurrences of 1 in $f$ is even, in which case $\psi(f)$ has an odd number of strict anti-excedances and an odd number of occurrences of 1 ,

(b) or $f$ has an odd number of strict anti-excedances and the number of occurrences of 1 in $f$ is odd, in which case $\psi(f)$ has an even number of strict anti-excedances and an even number of occurrences of 1.

The following theorem is a consequence of the above results.

Theorem 4.11 The appropriate restriction of the map $\psi$ is a bijection between the set of the subexceedant functions in $\mathcal{F}_{n}$ having image of cardinality $k$ with an odd number of occurrences of 1 and the set of subexceedant functions in $\mathcal{F}_{n}$ having image of cardinality $k$ with an even number of strict anti-excedances.

Corollary 4.12 The number $P_{n, k}$ equals the cardinality of the set of the subexceedant functions $f$ in $\mathcal{F}_{n}$ such that $\operatorname{IMA}(f)=k$ and $f$ contains an odd number of occurrences of 1 . The number $D_{n, k}$ equals of the set of the subexceedant functions $f$ in $\mathcal{F}_{n}$ such that $\operatorname{IMA}(f)=k$ and $f$ contains an even number of occurrences of 1.

\section{References}

[1] D. Dumont, Personal communication to the second author.

[2] D. Dumont, G. Viennot, A combinatorial interpretation of the Seidel generation of Genocchi numbers, Ann. Discrete Math. 6, 77-87, (1980).

[3] D. Foata, M. P. Schützenberger, Théorie géometrique des polynômes Eulériens, Lecture Notes in Mathematics, 138, Springer-Verlag, Berlin, (1970). 
[4] M. Hall, Proc. Sympos. Appl. Math. Combinatorial Analysis, Vol. 6, Amer. Math. Soc., Providence, R. I., p. 203 and following, (1956).

[5] D. Knuth, The art of computer programming, 3, Sorting and Searching, 2nd Ed., Addison-Wesley, Reading, (1981).

[6] D. H. Lehmer, Teaching combinatorial tricks to a computer, Proc. Sympos. Appl. Math. Combinatorial Analysis, Vol. 10, Amer. Math. Soc., Providence, R. I., 179-193, (1960).

[7] M. Lothaire, Combinatorics on words, Encyclopedia of Mathematics and Its Applications, Vol.17, AddisonWesley, Reading, MA, (1983).

[8] R. Mantaci, Sur la distribution des anti-excédances dans le groupe symétrique et dans ses sous-groupes, Theoret. Comp. Science A, 117, 243-253, (1993).

[9] R. Mantaci, Binomial coefficients and anti-excedances of even permutations: A Combinatorial Proof, Jour. of Comb. Theory, Series A,Vol.63, No. 2, pp 330-337, (July 1993).

[10] J. Riordan, An introduction to combinatorial analysis, New York, J. Wiley, (1958). 